OPEN ACCESS

Edited by:

Jamie F. Burr,

University of Guelph, Canada

Reviewed by:

Melissa L. Bates,

The University of lowa, United States Juan Manuel Murias,

University of Calgary, Canada

${ }^{*}$ Correspondence: Joana F. Reis

joana.reis@universidadeeuropeia.pt; joanareis@fmh.ulisboa.pt

Specialty section:

This article was submitted to

Exercise Physiology,

a section of the journal

Frontiers in Physiology

Received: 30 November 2018 Accepted: 25 March 2019

Published: 11 April 2019

Citation:

Reis JF, Fatela P, Mendonca GV, Vaz JR, Valamatos $M J$, Infante $J$, Mil-Homens P and Alves FB (2019)

Tissue Oxygenation in Response to Different Relative Levels of Blood-Flow Restricted Exercise. Front. Physiol. 10:407. doi: 10.3389/fphys.2019.00407

\section{Tissue Oxygenation in Response to Different Relative Levels of Blood-Flow Restricted Exercise}

\author{
Joana F. Reis ${ }^{1,2,3 *}$, Pedro Fatela ${ }^{3,4,5}$, Goncalo V. Mendonca ${ }^{2,4}$, Joao R. Vaz ${ }^{2,3,6}$, \\ Maria J. Valamatos ${ }^{2,4,5}$, Jorge Infante ${ }^{7}$, Pedro Mil-Homens ${ }^{2,4,5}$ and Francisco B. Alves ${ }^{1,2}$ \\ ${ }^{1}$ Laboratory of Physiology and Biochemistry of Exercise, Faculdade de Motricidade Humana, Universidade de Lisboa, \\ Lisbon, Portugal, ${ }^{2}$ Ciper, Faculdade de Motricidade Humana, Universidade de Lisboa, Lisbon, Portugal, ${ }^{3}$ Universidade \\ Europeia, Lisbon, Portugal, ${ }^{4}$ Neuromuscular Research Lab, Faculdade de Motricidade Humana, Universidade de Lisboa, \\ Lisbon, Portugal, ${ }^{5}$ Biomechanics and Functional Morphology Laboratory, Faculdade de Motrocidade Humana, Universidade \\ de Lisboa, Lisbon, Portugal, ${ }^{6}$ Department of Biomechanics, University of Nebraska at Omaha, Omaha, NE, United States, \\ ${ }^{7}$ Spertlab, Faculdade de Motricidade Humana, Universidade de Lisboa, Lisbon, Portugal
}

Blood flow restrictive (BFR) exercise elicits a localized hypoxic environment compatible with greater metabolic stress. We intended to compare the acute changes in muscle microvascular oxygenation following low-intensity knee extension exercise, combined with different levels of BFR. Thirteen active young men (age: $23.8 \pm 5.4$ years) were tested for unilateral knee extension exercise $(30+15+15+15$ reps at $20 \%$ one repetition maximum) on four different conditions: no-BFR (NOBFR), 40, 60, and $80 \%$ of arterial occlusion pressure (AOP). Deoxyhemoglobin+myoglobin concentration Deoxy[Hb+Mb], total hemoglobin [ $(\mathrm{H}+\mathrm{Hb})]$ and tissue oxygen saturation [TOI] were measured on the vastus lateralis muscle using near-infrared spectroscopy (NIMO, Nirox srl, Brescia, Italy). The magnitude of change in Deoxy[Hb+Mb]during exercise was similar between 60 and 80\% AOP. Overall, compared to that seen during 60 and $80 \%$ AOP, NOBFR as well as 40\% AOP resulted in a lower magnitude of change in Deoxy[Hb+Mb] $(p<0.05)$. While the oxygen extraction decreased during each interset resting interval in NOBFR and 40\% AOP, this was not the case for 60 or $80 \%$ AOP. Additionally, TOI values obtained during recovery from each set of exercise were similarly affected by all conditions. Finally, our data also show that, when performed at higher restrictive values (60 and $80 \%$ ), BFR exercise increases total Deoxy[Hb+Mb] extraction ( $p<0.05)$. Taken together, we provide evidence that BFR is effective for increasing deoxygenation and reducing tissue oxygenation during low-intensity exercise. We also showed that when using low loads, a relative pressure above $40 \%$ of the AOP at rest is required to elicit changes in microvascular oxygenation compared with the same exercise with unrestricted conditions.

Keywords: muscle oxygenation, KAATSU, resistance exercise, near-infrared spectroscopy, oxygen extraction

\section{INTRODUCTION}

The chronic effect of using tourniquet cuffs to restrict muscle blood flow during resistance exercise on muscle size and strength are well established in the available literature (Loenneke et al., 2012; Slysz et al., 2016). Despite significant efforts to fully comprehend the physiological basis of muscle growth resulting from blood flow restricted (BFR) exercise, the specific mechanisms underlying such response remain largely unknown (Manini and Clark, 2009). There is general 
agreement that muscle hypertrophy is triggered by the combined effect of mechanical tension, muscle damage and metabolic stress (Schoenfeld, 2010). Several studies have shown that performing BFR exercise with $20 \%$ of $1 \mathrm{RM}$ induces the same or even greater fatiguing stimulus when compared with high intensity exercise training (Takarada et al., 2000; Cook et al., 2007). Thus, it can be used in multiple exercise settings, particularly when high intensity resistance exercise is not recommended (Cook et al., 2007). Since BFR exercise is typically performed using light loads, metabolic stress is believed to be largely responsible for enhancements in both muscle size and strength after training (Takarada et al., 2000, 2002).

Past research has shown that microvascular oxygenation varies as a function of BFR absolute pressure and duration (Karabulut et al., 2014; Neto et al., 2014). Nevertheless, it is important to note that BFR exercise is typically structured using a predetermined number of sets and repetitions and that, within this context, the magnitude of neuromuscular activation and fatigue varies as a function of BFR relative pressure (Fatela et al., 2016). For this reason, several reports focused on exploring the overall impact (i.e., during and between sets) of BFR on microvascular oxygenation during low-intensity resistance exercise (Ganesan et al., 2015; Lauver et al., 2017; Yanagisawa and Sanomura, 2017). Their findings are somewhat conflicting, and this is probably a consequence of differences between methodological designs (e.g., exercise selection, BFR level as well as duration, contraction mode and site for monitoring tissue oxygenation). To our knowledge, only one previous investigation examined the role of relative BFR pressure on microvascular oxygenation (Kilgas et al., 2018). Using handgrip exercise, it was shown that setting BFR to 60 and $80 \%$ of the pressure required to block arterial blood flow, or arterial occlusion pressure (AOP) elicited a reduction in tissue saturation index and an increase in deoxyhemoglobin+myoglobin concentration $(\mathrm{Deoxy}[\mathrm{Hb}+\mathrm{Mb}])$. However, due to the characteristics of this specific exercise paradigm, these findings may not be extensive to dynamic exercise involving larger muscle mass. Determining the optimal level of BFR pressure, relative to AOP, is fundamental for maximizing the effectiveness of exercise prescription because it might influence the possible mechanism for the adaptations following BFR training (Cayot et al., 2014; Scott et al., 2015).

Therefore, we aimed at comparing the acute response of Deoxy $[\mathrm{Hb}+\mathrm{Mb}]$, total hemoglobin + myoglobin $[\mathrm{T}(\mathrm{H}+\mathrm{Mb})]$ and tissue oxygen saturation (TOI) in the vastus lateralis muscle during low-intensity exercise performed at different levels of relative $B F R$. We hypothesized that, during dynamic knee extension exercise, the peripheral deoxygenation would increase, and total hemoglobin + myoglobin would increase as a function of the percent arterial occlusion pressure.

\section{MATERIALS AND METHODS}

\section{Participants}

Thirteen active young men (age: $23.8 \pm 5.4$ years; height: $174.8 \pm 4.2 \mathrm{~cm}$; body mass: $69.8 \pm 7.0 \mathrm{~kg}$; Systolic blood pressure:
$122.6 \pm 7.0 \mathrm{mmHg}$; diastolic blood pressure: $76.7 \pm 8.5 \mathrm{mmHg}$; AOP: $136.6 \pm 9.3 \mathrm{mmHg}$ ) volunteered to participate in this study. The participants were fully informed of any risk and discomfort associated with the experiments before providing written consent. Participants were not enrolled in any kind of resistance or endurance training in the 6 months prior to the participation in the study. They were all non-smokers and free from any known cardiovascular and metabolic diseases, as assessed by medical history. The participants were instructed to maintain the same level of physical activity throughout the course of the study. They were asked to avoid exercise as well as the intake of caffeine and alcohol for at least $24 \mathrm{~h}$ before testing. Testing was performed at the same time of day for standardization purposes with at least $48 \mathrm{~h}$ between testing sessions. This study was approved by the Faculty's Ethics Committee (CEFMH 17/2014) and in accordance with the Declaration of Helsinki.

\section{Experimental Design}

All participants were familiarized with the testing procedures in two separate sessions. Metronome pacing and AOP measurements were performed during the first session. Then, on a different day, all participants performed a baseline session, where (a) AOP for the right lower limb was reassessed, (b) one repetition maximum (1RM) was determined for the right knee extensors and (c) testing (including metronomic pacing) was again reproduced. Using a crossover design, participants then visited the laboratory on four additional days to complete the following resistance exercise trials in a randomized order: without BFR (NOBFR), 40, 60, and 80\% AOP. Participants were tested in the seated position and were fixed with chest and abdominal straps. Vascular restriction was elicited using a $13 \times 124 \mathrm{~cm}$ pneumatic cuff (SC12L Tourniquet Cuffs, D. E. Hokanson, Inc., Bellevue, WA), applied to the most proximal portion of the right thigh and the pressure was maintained throughout the test.

The exercise protocol was performed using an isokinetic dynamometer (Biodex System 3, Biodex Medical Systems, Shirley, NY), and consisted of a series of unilateral knee extensions at $20 \%$ of $1 \mathrm{RM}$ throughout all testing sessions. This training intensity was selected because of its effectiveness in inducing fatigue when combined with BFR (Takarada et al., 2000; Cook et al., 2007). During exercise, participants were monitored for microvascular oxygenation using near-infrared spectroscopy (NIRS) (NIMO, Nirox srl, Brescia, Italy). Knee-extension peak torque was also determined during maximal voluntary isometric contraction (MVIC) before and after acute exercise.

\section{Determination of AOP}

Arterial occlusion pressure was determined using a vascular Doppler probe (PD1+ Combi, Ultrasound Technologies Ltd., Caldicot, United Kingdom) placed over the right posterior tibial artery, halfway between the posterior border of the medial malleolus and the Achilles tendon. A pneumatic cuff was inflated (E20/AG101 Rapid Cuff Inflator) gradually up to the point when the auscultatory pulse of the posterior tibial artery was interrupted (Fatela et al., 2018). To guarantee similar 
cuff placement between familiarization and testing sessions, a photographic record was made for each participant.

\section{RM Testing}

The maximal torque produced in a single repetition was determined for the right knee extension using the isotonic mode of the isokinetic dynamometer. The participants were asked to complete one repetition through a full range of motion $\left(90^{\circ}\right)$. Strong verbal encouragement was given in each trial, and $2 \mathrm{~min}$ of recovery were allowed between attempts. 1RM was always determined within five trials (mean value: $219.2 \pm 37.8 \mathrm{Nm}$ ).

\section{Maximal Voluntary Isometric Contraction}

Maximal voluntary isometric contraction was determined at the optimal joint angle for right knee extension in all testing sessions. Participants performed three isometric knee extension trials $(3 \mathrm{~s}$ per trial). They were instructed to exert their maximum force as fast and hard as possible. One min of recovery between trials was allowed. Peak torque was set as pre- and post-exercise MVIC ( 2 min post-cuff deflation).

\section{Exercise Protocol}

After a standardized warm-up (6 min of unloaded cycleergometry), participants performed 4 sets of knee extension at $20 \% 1 \mathrm{RM}(30+15+15+15$ reps, set $1-4$, respectively), with $30 \mathrm{~s}$ of passive rest between sets (rest 1-3) (Yasuda et al., 2008; Loenneke et al., 2013). A metronome was used to control the concentric-concentric mode, with $1 \mathrm{~s}$ for knee extension (20\% 1-RM) and another for knee flexion (unloaded). Verbal encouragement was provided to warrant that each participant completed the full exercise protocol. For safety reasons, a pulse oximeter (Onyx ${ }^{\otimes}$ II 9560, Nonin Medical Inc., Plymouth, MN) was placed in the right hallux, immediately after each set to ensure that blood flow was not completely halted by tissue edema. In the BFR sessions, the cuff was inflated before exercise.

\section{NIRS Signal}

Near-infrared spectroscopy provides non-invasive information about the changes in oxygenation and hemodynamics in muscle tissue based on the oxygen-dependent characteristics of nearinfrared light (Perrey and Ferrari, 2018) and has been validated for multiple forms of resistance exercise (Pereira et al., 2007). NIRS measurements were performed on the vastus lateralis muscle of the right leg throughout the entire duration of the exercise protocol. The skin of each participant's right leg was initially cleaned and shaved. Then, the probe was placed on the belly of the muscle, midway between the lateral epicondyle and greater trochanter of the femur. The placement of the probe was marked with indelible ink and a photographic recording was taken to ensure similar placement of the probe between sessions. The probe was attached to the skin surface with tape and then covered with an optically dense elastic bandage. This minimized its movement and prevented the intrusion of extraneous light and loss of near NIR-transmitted light from the field of interrogation.

Deoxy $[\mathrm{Hb}+\mathrm{Mb}], \quad$ oxy hemoglobin + myoglobin $\left[(\mathrm{Hb}+\mathrm{Mb}) \mathrm{O}_{2}\right]$ and $[\mathrm{T}(\mathrm{Hb}+\mathrm{Mb})]$ were quantified with a continuous-wave tissue oximeter (NIMO, Nirox srl, Brescia, Italy). TOI was expressed in $\%$ and calculated as:

$$
[(H b+M b) O 2] /[(H b+M b) O 2]+D e o x y[H b+M b] \times 100 .
$$

Briefly, this system is based on the $\mathrm{O}_{2}$ dependency of absorption changes for near infra-red light in hemoglobin and myoglobin and it consists on an emission probe which emits three wave lengths $(685,850$, and $980 \mathrm{~nm})$ and a photon detector. The intensity of incident and transmitted light was recorded continuously at $40 \mathrm{~Hz}$ and used to estimate Deoxy[Hb+Mb], (1) $[(\mathrm{Hb}+\mathrm{Mb}) \mathrm{O} 2]$ and $[\mathrm{T}(\mathrm{Hb}+\mathrm{Mb})]$ (Rovati et al., 2004; Ferrari et al., 2011). As the NIRS signal is unable to distinguish between hemoglobin and myoglobin, results are considered as the combined $\mathrm{O}_{2}$ saturation of these metalloproteins. To account for the possible influence of the local fat layer on NIRS, a realtime correction using an algorithm included in the software (Nimo Data Analysis Peak) was used. Deoxy[Hb+Mb]signal is less dependent of changes in blood flow and is considered as an indicator of fractional $\mathrm{O}_{2}$ extraction within the microvascular level (Ferrari et al., 1997). [T(Hb+Mb)] reflects the total amount of hemoglobin, and it can be interpreted as changes in blood volume within the tissue vascular beds (Van Beekvelt et al., 2001). TOI reflects the dynamic balance between $\mathrm{O}_{2}$ supply and $\mathrm{O}_{2}$ uptake and is independent of near-infrared photon path length in muscle tissue (Brocherie et al., 2015).

\section{Data Handling}

Baseline values for each testing session were established as 1-min averages for NIRS derived signal. To minimize the impact of baseline differences between trials, Deoxy[Hb+Mb] and $[\mathrm{T}(\mathrm{Hb}+\mathrm{Mb})]$ values were computed as changes $(\Delta)$ from baseline within each testing session. Deoxy $[\mathrm{Hb}+\mathrm{Mb}]$ and $[\mathrm{T}(\mathrm{Hb}+\mathrm{Mb})]$ and TOI were measured throughout the exercise protocol. For these analyses, sets were divided into thirds and mean value \pm SD of the last third was used as representative of each correspondent phase. (Set 1, Recovery 1, Set 2, Recovery 2, Set 3, Recovery 3 , Set 4$). \Delta[T(H b+M b)]$ was also analyzed for the recovery periods.

Relative recovery (\%Rec) for Deoxy $[\mathrm{Hb}+\mathrm{Mb}]$ and $[\mathrm{T}(\mathrm{Hb}+\mathrm{Mb})]$ and TOI was determined as the \% difference between the last third of recovery and the last third of the previous set. Total oxygen extraction was computed as the area under the Deoxy $[\mathrm{Hb}+\mathrm{Mb}]$ curve including all sets and recovery periods (Soares et al., 2017).

\section{Statistical Analysis}

Data are reported as mean \pm SD, unless otherwise specified. The Mauchly's test was used to test the assumption of sphericity. The Greenhouse-Geisser correction was implemented to adjust the degrees of freedom for the averaged tests of significance when the assumption of sphericity was not met. Paired $t$-tests were used for exploring possible differences in peak torque from pre- to post-exercise at each condition (NOBFR, 40,60 , and $80 \%$ AOP). A two-way repeated measures ANOVA [four conditions (NOBFR vs. 40 vs. 60 vs. $80 \% \mathrm{AOP}$ ) $\times 5$ times (Pre-exercise vs. set 1 vs. set 2 vs. set 3 vs. set 4 )] was used 
to determine the impact of BFR on each dependent variable (i.e., Deoxy[Hb+Mb] and $[\mathrm{T}(\mathrm{Hb}+\mathrm{Mb})]$ and TOI). Another twoway repeated measures ANOVA [four conditions (NOBFR vs. 40 vs. 60 vs. $80 \% \mathrm{AOP}$ ) $\times 3$ times (recovery 1 vs. recovery 2 vs. recovery 3)] was additionally used to determine the impact of each condition on the \%Rec of Deoxy[Hb+Mb] and $[\mathrm{T}(\mathrm{Hb}+\mathrm{Mb})]$ and TOI. When a significant effect was detected at a significance level of $p<0.05, t$-tests were used for post hoc comparisons. Adjustment for multiple comparisons was made with Bonferroni's correction. All statistical calculations were computed using the Statistical Package for the Social Sciences (SPSS) version 25.0 (SPSS Inc., Chicago, IL). Significance was set at $p<0.05$.

\section{RESULTS}

Peak torque was similar between pre- and post-exercise time points in all conditions, except for $80 \%$ AOP (reduction of 5.8\%, $p<0.05)$. Tables $\mathbf{1 - 3}$ show the changes of Deoxy $[\mathrm{Hb}+\mathrm{Mb}]$ and $[\mathrm{T}(\mathrm{Hb}+\mathrm{Mb})]$ and TOI in transition from baseline to exercise (sets 1-4). We obtained a conditionby-time interaction for Deoxy[Hb+Mb] and $[\mathrm{T}(\mathrm{Hb}+\mathrm{Mb})]$ ( $F=12.4$ and 10.3, $p<0.05$; respectively). Post hoc analyses indicate that $[\mathrm{HHb}]$ increased from pre-exercise to set 1 in both NOBFR and 40\% AOP $(p<0.05)$. No other changes were noted for this parameter during exercise. With the exception of pre-exercise, both these conditions exhibited similar Deoxy $[\mathrm{Hb}+\mathrm{Mb}]$ values over time. As importantly, Deoxy $[\mathrm{Hb}+\mathrm{Mb}]$ remained unchanged over time during exercise performed at 60 and $80 \%$ AOP. Nevertheless, Deoxy[Hb+Mb] values were consistently lower during NOBFR and 40\% AOP when compared to that seen at 60 and $80 \%$ AOP throughout all time points $(p<0.05)$. Finally, the acute response of Deoxy $[\mathrm{Hb}+\mathrm{Mb}]$ to low-intensity exercise was similar between 60 and $80 \%$ AOP.

$[\mathrm{T}(\mathrm{Hb}+\mathrm{Mb})]$ decreased with BFR exercise $(p<0.05)$ and then remained stable after set 1 . Conversely, no changes were seen in $[\mathrm{T}(\mathrm{Hb}+\mathrm{Mb})]$ in response to NOBFR exercise. There were significant differences in pre-exercise $[\mathrm{T}(\mathrm{Hb}+\mathrm{Mb})]$ between NOBFR, AOP 40 and 60\% ( $p<0.05)$. Conversely, this was not the case for comparisons between AOP 60 and 80\%. Even though, all conditions attained similar endpoints at the completion of set 1 and $4,[\mathrm{~T}(\mathrm{Hb}+\mathrm{Mb})]$ was higher in 60 vs. $40 \%$ AOP after set 2 and $3(p<0.05)$. In Set $3,[\mathrm{~T}(\mathrm{Hb}+\mathrm{Mb})]$ was also increased following exercise with $60 \%$ AOP vs. NOBFR $(p<0.05)$.

There were significant main effects of condition and time for TOI $(F=4.3$ and 63.0, respectively; $p<0.05$ ). Follow up analyses revealed that TOI was lower in $80 \%$ AOP than in NOBFR $(p<0.05)$. No other differences were seen between conditions. Additionally, TOI decreased in all conditions from pre-exercise to set $1(p<0.05)$ and then remained stable until the end of set 4 .

TABLE 1 | Delta changes in deoxygenated hemoglobin+myoglobin (AU) (Mean and \pm standard deviation) for before exercise (Pre) and Set $1-4$ in each blood flow restrictive pressure.

\begin{tabular}{|c|c|c|c|c|c|}
\hline & Pre & Set 1 & Set 2 & Set 3 & Set 4 \\
\hline NOBFR & $-2.3 \pm 4.9$ & $19.4 \pm 8.8^{a}$ & $18.9 \pm 11.0$ & $19.3 \pm 11.3$ & $19.5 \pm 11.2$ \\
\hline $40 \% \mathrm{AOP}$ & $11.0 \pm 4.8^{*}$ & $21.2 \pm 8.8^{a}$ & $20.0 \pm 8.3$ & $19.9 \pm 8.6$ & $20.2 \pm 8.4$ \\
\hline $60 \%$ AOP & $18.7 \pm 10.9 * \S$ & $27.3 \pm 10.9 * \S$ & $27.6 \pm 11.5^{* \S}$ & $27.8 \pm 12.0 * \S$ & $27.3 \pm 11.8^{* \S}$ \\
\hline $80 \%$ AOP & $25.6 \pm 10.9 * \S$ & $27.3 \pm 12.1^{* \S}$ & $27.7 \pm 12.3^{*} \S$ & $27.2 \pm 12.6$ & $27.5 \pm 12.4^{* \S}$ \\
\hline
\end{tabular}

* Significantly different from NOBFR; § Significantly different from 40\% AOP; a Significantly different from PRE; (p < 0.05).

TABLE 2 | Delta changes in total hemoglobin+myoglobin (AU) (Mean and \pm standard deviation) for before exercise (Pre) and Set 1-4 in each blood flow restrictive pressure.

\begin{tabular}{|c|c|c|c|c|c|}
\hline & Pre & Set 1 & Set 2 & Set 3 & Set 4 \\
\hline NOBFR & $-2.5 \pm 13.2$ & $-5.0 \pm 14.9$ & $-3.7 \pm 15.3$ & $-6.3 \pm 16.6$ & $-3.8 \pm 18.1$ \\
\hline $40 \%$ AOP & $24.1 \pm 15.0^{*}$ & $-4.6 \pm 16.6^{a}$ & $-4.3 \pm 14.2$ & $-4.4 \pm 18.6$ & $-0.8 \pm 17.8$ \\
\hline $60 \%$ AOP & $45.1 \pm 24.6^{* \S}$ & $6.8 \pm 14.1^{a}$ & $12.4 \pm 14.0^{\S}$ & $13.2 \pm 14.6^{*}$ & $13.5 \pm 18.0$ \\
\hline $80 \%$ AOP & $48.5 \pm 23.0^{* \S}$ & $-0.8 \pm 18.7^{a}$ & $8.5 \pm 14.5$ & $6.6 \pm 15.8$ & $12.3 \pm 13.4$ \\
\hline
\end{tabular}

* Significantly different from NOBFR; $§$ Significantly different from 40\% AOP; a significantly different from PRE; (p < 0.05).

TABLE 3 | Tissue oxygenation index (AU) (Mean and \pm standard deviation) before exercise (Pre) and Set 1-4 in each blood flow restrictive pressure.

\begin{tabular}{|c|c|c|c|c|c|}
\hline & Pre & Set 1 & Set 2 & Set 3 & Set 4 \\
\hline NOBFR & $80.6 \pm 3.5$ & $65.2 \pm 9.5^{a}$ & $65.9 \pm 11.1$ & $65.0 \pm 11.1$ & $65.6 \pm 10.2$ \\
\hline $40 \%$ AOP & $72.7 \pm 7.4$ & $58.1 \pm 13.9^{a}$ & $59.0 \pm 14.4$ & $59.1 \pm 14.4$ & $60.4 \pm 12.7$ \\
\hline $60 \%$ AOP & $73.1 \pm 7.5$ & $58.9 \pm 14.2^{a}$ & $60.5 \pm 13.6$ & $61.0 \pm 12.6$ & $61.5 \pm 12.8$ \\
\hline $80 \%$ AOP & $69.7 \pm 8.9$ & $55.6 \pm 13.8^{a}$ & $58.5 \pm 13.1$ & $58.3 \pm 13.3$ & $59.9 \pm 11.9^{*}$ \\
\hline
\end{tabular}

* Significantly different from NOBFR; ${ }^{a}$ significantly different from PRE; $(p<0.05)$. 
During the inter-set recovery intervals, the relative changes from baseline in $[\mathrm{T}(\mathrm{Hb}+\mathrm{Mb})]$ presented a significant main effect for condition $(F=21.7 ; p<0.01)$. Follow up analyses revealed that this variable increased progressively with the restrictive pressures. However, there were no significant differences between 60 and $80 \%$ AOP (Table 4).

As depicted in Figure 1, depending on the condition, there were different levels of muscle oxygenation during each interset recovery period. Specifically, while Deoxy[Hb+Mb] exhibited a high level of recovery following each NOBFR and $40 \%$ AOP exercise set, this was not the case for 60 nor $80 \%$ AOP $(p<0.05)$. There was virtually no recovery in Deoxy[Hb+Mb] after $60 \%$ $\mathrm{AOP}$ and, in $80 \% \mathrm{AOP}$, the level of oxygen extraction actually increased. TOI exhibited a significant recovery in all conditions during the inter-set periods $(p<0.05)$. The magnitude of TOI recovery was similar between NOBFR and $40 \% \mathrm{AOP}$ and between 60 and $80 \%$ AOP, respectively (Figure 2). [T( $\mathrm{Hb}+\mathrm{Mb})]$ showed no significant differences between conditions for the percentage of recovery from each set.

The area under the Deoxy $[\mathrm{Hb}+\mathrm{Mb}]$ curve was used to explore the impact of each exercise protocol on total oxygen extraction. As can be seen in Figure 3, the area under the Deoxy[Hb+Mb] curve increased as a function of the percentual AOP pressure used $(p<0.05)$. However, it should be noted that the differences in this parameter did not achieve significance for comparisons between 60 and $80 \%$ AOP $(p>0.05)$.

TABLE 4 | Delta changes in total hemoglobin+myoglobin (AU) (Mean and \pm standard deviation) for Recovery 1-3 in each blood flow restrictive pressure.

\begin{tabular}{lrrc}
\hline & Recovery $\mathbf{1}$ & Recovery $\mathbf{2}$ & \multicolumn{1}{c}{ Recovery $\mathbf{3}$} \\
\hline NOBFR & $6.6 \pm 10.3$ & $4.3 \pm 13.8$ & $4.1 \pm 16.2$ \\
$40 \%$ AOP & $26.8 \pm 13.2$ & $25.0 \pm 17.8$ & $25.1 \pm 20.5^{*}$ \\
$60 \%$ AOP & $43.8 \pm 13.0$ & $41.7 \pm 12.0$ & $43.4 \pm 15.7$ * \\
$80 \%$ AOP & $41.5 \pm 17.6$ & $40.9 \pm 15.3$ & $45.2 \pm 16.9 * \S$
\end{tabular}

*Significantly different from NOBFR; $\S$ Significantly different from $40 \%$ $A O P(p<0.05)$.

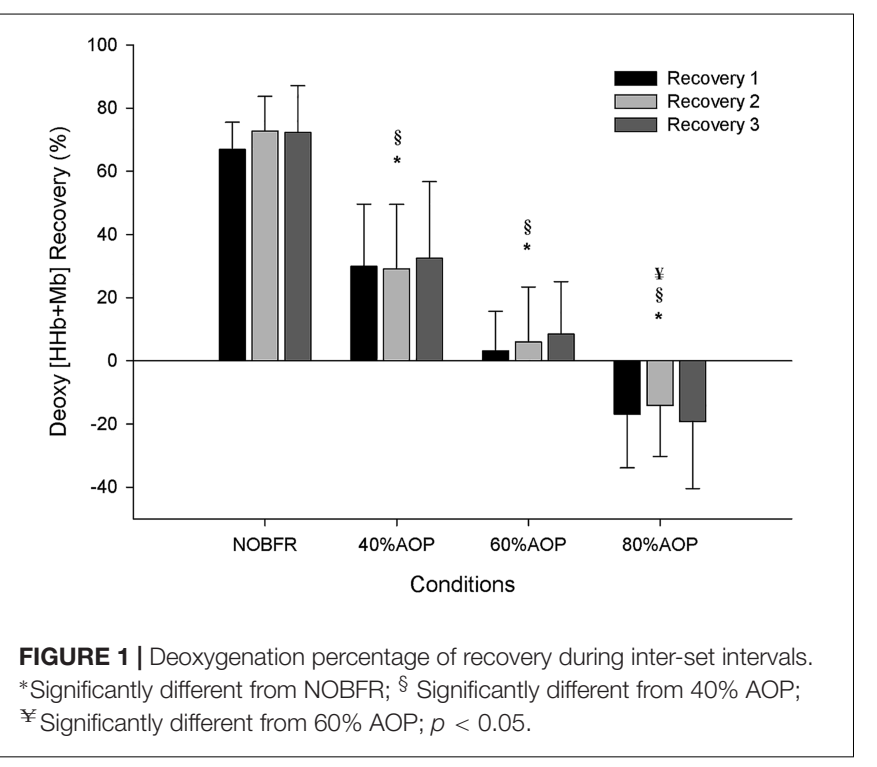

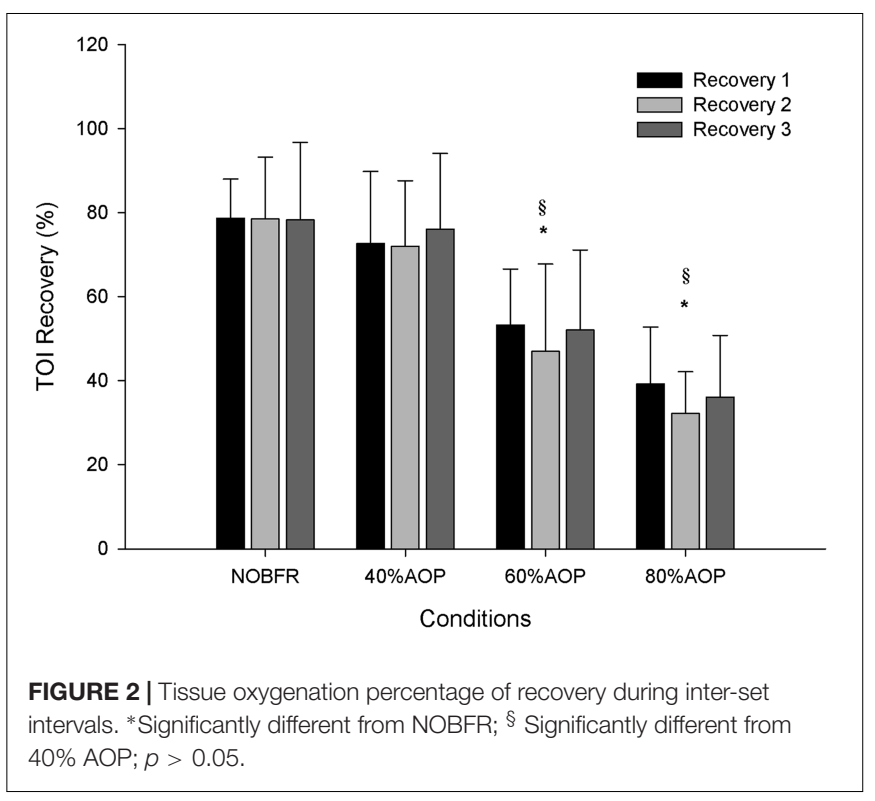

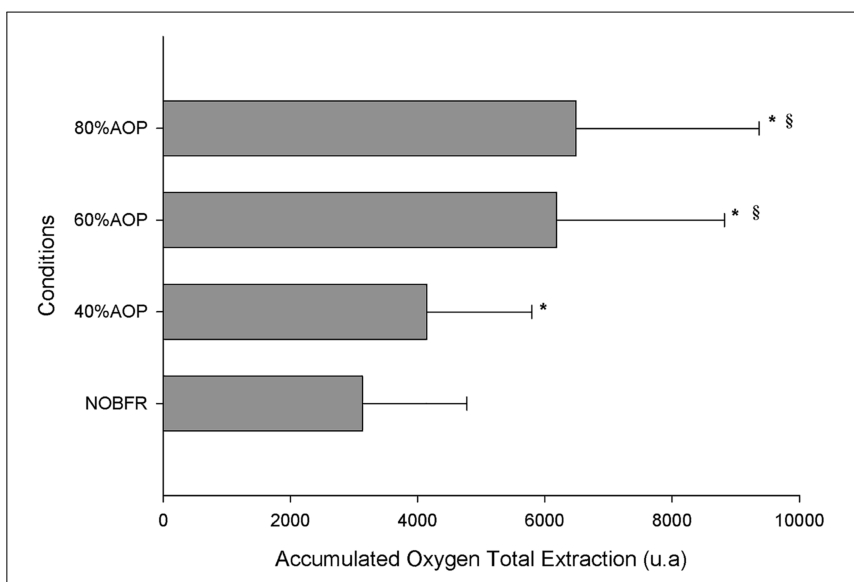

FIGURE 3 | Total oxygen extraction represented as accumulated change from baseline in Deoxy[Hb+Mb] *Significantly different from NOBFRE; $§$

Significantly different from 40\% AOP; $p>0.05$.

\section{DISCUSSION}

We explored the interaction between different levels of BFR, relative to $\mathrm{AOP}$, and muscle oxygenation in response to an acute multi-set knee-extension exercise protocol. It was demonstrated that BFR increases deoxygenation and hampers tissue oxygenation during low-intensity muscle contractions. Thus, our hypotheses were partially confirmed. However, we also showed that to induce a considerable stress on microvascular oxygenation, represented by increased deoxygenation levels, using low loads (20\% RM), BFR should be set above $40 \%$ of AOP. Otherwise, the level of muscle oxygenation/deoxygenation is not substantially different from that seen during non-BFR exercise. Since we did not test pressures between 40 and $60 \%$ AOP, we can only say that in the three pressures studied, $60 \%$ AOP appears to represent a threshold required to induce higher 
deoxygenation and decreased tissue oxygenation levels within this form of resistance training. As importantly, we provide evidence that setting BFR to $80 \%$ of AOP exerts no further impact in altering microvascular deoxygenation compared to that seen using $60 \%$. This is important because it corroborates past findings on BFR-induced muscle fatigue and activation (Fatela et al., 2016).

The use of different restrictive pressures interacts with muscle activation and neuromuscular fatigue (Loenneke et al., 2015; Fatela et al., 2016). According to our findings, this is also the case for microvascular oxygenation during acute lowintensity BFR exercise. BFR is thought to reduce venous return, while eliciting a turbulent flow in the arterial circulation. Ultimately, this decreases blood flow velocity within the tissues distal to the cuff (Manini and Clark, 2009). There is general agreement that the acute impact of BFR induces local hypoxia as well as an accumulation of metabolites (resulting from increased production and limited removal) (Scott et al., 2015). Our findings of increased deoxygenation and lower tissue $\mathrm{O}_{2}$ saturation, obtained during exercise with 60 and $80 \%$ AOP, further support this concept. This is the first study focusing on the interaction between BFR and tissue oxygenation, using a typical and well-validated exercise protocol (Loenneke et al., 2014a; Scott et al., 2015). Past reports have used NIRS measurements to examine muscle oxygenation during handgrip, eccentric, isometric or limited volume exercise as well as in response to muscle contractions performed to failure (Cayot et al., 2014; Ganesan et al., 2015; Lauver et al., 2017; Yanagisawa and Sanomura, 2017; Kilgas et al., 2018). Accordingly, comparisons between our results and those of the available research are challenging. Nevertheless, using an exercise protocol combining CON and ECC muscle contractions, Lauver et al. (2017), also showed heightened deoxygenation with BFR set at $130 \%$ of resting systolic blood pressure. Similar findings were also reported for low-volume isometric exercise, combined with BFR set at 60 and $80 \%$ of AOP (Cayot et al., 2014). Conversely, Ganesan et al. (2015) did not find a higher muscle oxygen extraction in BFRE knee extension exercise sets when compared with time matched unrestricted exercise. However, these authors prescribed an absolute and arbitrary BFR value of $100 \mathrm{~mm} \mathrm{Hg}$, which most likely affected their findings.

In our study, the differences observed in the Deoxy $[\mathrm{Hb}+\mathrm{Mb}]$ response were noticeable before the beginning of exercise a time point representing the effect of BFR on microvascular oxygenation per se. Using 60 and $80 \%$ AOP resulted in higher values of Deoxy[Hb+Mb] throughout the testing protocol. While Kilgas et al. (2018) showed a progressive increase in Deoxy $[\mathrm{Hb}+\mathrm{Mb}]$ during exercise set at 60 and $80 \%$ AOP, we found that, under these specific circumstances, deoxy[Hb+Mb] remained unaltered from pre-exercise to the end of each set. Such discrepancies might be secondary to the differences between studies in the total length of time exposure to BFR (longer in our study). As importantly, Kilgas et al. (2018) analyzed only 1 set of isolated handgrip exercise for each restrictive pressure and all participants exercised using several levels of BFR within the same day. Thus, data from this study are most likely contaminated by the BFRassociated reactive hyperaemia (Mouser et al., 2017). However, we must acknowledge that we did not establish the level of venous return restriction induced by the different restrictive pressures. Therefore, we cannot establish on what extent the increased extraction in 60 and $80 \%$ AOP was due to the limited venous return.

We calculated the accumulated Deoxy[Hb+Mb] variation across the total exercise protocol. This represents the total oxygen extraction occurring during both exercise and rest periods (Soares et al., 2017). We found that the accumulated $\mathrm{Deoxy}[\mathrm{Hb}+\mathrm{Mb}]$ increased in parallel with BFR relative pressure. However, there were no differences between the impact of 60 and $80 \%$ AOP. Although we did not measure the pressure required to occlude venous return, we speculate that above $60 \%$ AOP there was no added effect; thus explaining the plateau on deoxy $[\mathrm{HHb}+\mathrm{Mb}]$. In fact, previous research shows that venous occlusion occurs at somewhat lower pressures. For example, it may be induced at an absolute pressure of $60 \mathrm{mmHg}$ or even at $20 \mathrm{mmHg}$ below diastolic blood pressure. Moreover, it has also been shown that BFR pressures between 45 and $200 \mathrm{mmHg}$ produce a similar effect on reducing left ventricular end-diastolic volume - thus corroborating the concept that venous occlusion is attained at low levels of cuff pressure (Iida et al., 2005; Corrigan et al., 2008). In support of this, in most studies focusing on measuring blood flow to the lower limb using plethysmography, $50 \mathrm{mmHg}$ is generally used as effective for inducing total venous occlusion (Patterson and Ferguson, 2010), and this is considerably lower than the pressure used at $60 \% \mathrm{AOP}$. In fact, the pressure that we used to induce $60 \% \mathrm{AOP}$ was $>$ than $50 \mathrm{mmHg}$ and $>$ than diastolic blood pressure. Conceptually, this confirms that there is no added restriction of venous return between 60 and $80 \%$ AOP. Additionally, we found that in the recovery periods between sets, the two higher pressures presented significantly higher values of $\mathrm{T}[\mathrm{HHb}+\mathrm{Mb}]$, which can be considered a proxy for blood flow. However, there were no differences between 60 and $80 \%$ AOP in this variable.

It has been hypothesized that the optimal BFR pressure may follow a hormetic-like relationship (Loenneke et al., 2014b; Scott et al., 2015; Kilgas et al., 2018), with some literature postulating that high pressures may not enhance muscular development more than moderate pressures. Even though recent data suggest that BFR exercise protocols may benefit from higher levels of restriction (80\%) when exercising at very low intensities (Lixandrão et al., 2015), the authors only compared the effects of 12 weeks of training with either 40 or $80 \%$ AOP. Our data are in accordance with those of Kilgas et al. (2018) for arm exercise in that $60 \%$ AOP might represent a physiological threshold for tissue hypoxia and metabolite accumulation during low-intensity exercise.

$[\mathrm{T}(\mathrm{Hb}+\mathrm{Mb})]$, which is considered as a surrogate index of changes in tissue blood volume (Grassi et al., 1999; Van Beekvelt et al., 2001; Perrey and Ferrari, 2018), was also influenced by different levels of restrictive pressures before exercise. Specifically, heightened restrictive pressures induced 
progressive increases in $[\mathrm{T}(\mathrm{Hb}+\mathrm{Mb})]$, which can be attributed to the venous pooling distal to the cuff (Pope et al., 2013; Lauver et al., 2017). However, at a BFR level $>$ than $60 \%$ of AOP we observed no further increase in $[\mathrm{T}(\mathrm{Hb}+\mathrm{Mb})]$, which could result from similar degrees of venous return constrains in these two restrictive pressures. However, it is important to note that $80 \%$ AOP was the only condition compatible with a lower TOI and a small, but significant decrease in peak torque post-exercise. Since Deoxy $[\mathrm{Hb}+\mathrm{Mb}]$ and $[\mathrm{T}(\mathrm{Hb}+\mathrm{Mb})]$ were similar between 60 and $80 \%$ AOP, the greater magnitude of reduction in TOI values at higher levels of relative restriction was most likely caused by further reductions in arterial inflow.

In contrast to that seen during exercise, the inter-set recovery periods were characterized by considerable differences between conditions even at higher percentual of AOP. Globally, our data indicate that recovery of muscle deoxygenation resulting from each set of contractions varies depending on the magnitude of restrictive pressure. Specifically, while the relative recovery of Deoxy[Hb+Mb] was similar between NOBFR and 40\% AOP, considerable differences were obtained when comparing 60 to $80 \%$ AOP. Specifically, we found that after low-intensity exercise performed at 60\% AOP, Deoxy[Hb+Mb] does not recovery after $30 \mathrm{~s}$ of pause between sets. Conversely, when exercising at $80 \%$ AOP, muscle deoxygenation was actually potentiated during each inter-set rest interval. Although beyond the scope of this study, we speculate that this might be secondary to enhancements in the $\mathrm{O}_{2}$ deficit resulting from exercise performed at higher levels of BFR (Mendonca et al., 2015; Conceição et al., 2018).

According to the existent literature, the metabolic stress induced from multiple sets of BFR exercise is similar to that seen during high-intensity exercise, but only if the cuff inflation is maintained during recovery from each set (Suga et al., 2012). This approach ensures heightened muscle activation and maximizes training adaptations, independently of the external load (Loenneke et al., 2011). Although we did not compare this setting with a protocol where the cuff was release between sets, our results seems to be in line with the literature. However, we showed that all conditions were compatible with some recovery in tissue oxygenation during each inter-set period. Yet, there was a more pronounced recovery of TOI in NOBFR and 40\% AOP compared to that seen during 60 and $80 \%$ AOP. Thus, we contend that, for ensuring metabolite buildup during exercise, the cuff should be kept inflated at a level $\geq 60 \%$ AOP.

\section{Limitations}

There are several limitations in this study. For instance, the contribution of myoglobin desaturation is not possible to determine via NIRS. Thus, the differences between blood-muscle oxygen transport are not distinguishable in the current study (Ferrari et al., 2011). Additionally, NIRS signal is influenced by the thickness of adipose tissue and by the effect of changes in blood volume along within the tissue (Ferrari et al., 2004). Furthermore, even though we took precautionary measures to improve the reproducibility of probe placement (i.e., using indelible ink and taking photographic records), the day-today variation of these specific parameters corresponds to $\sim$ 8.0-9.4\% (Willis et al., 2017). Nevertheless, we are confident that our data were not affected by this, because the use of relative changes in the NIRS variables, which is common in the research area, allows valid comparisons between settings (Perrey and Ferrari, 2018).

We did not establish the venous return occlusion pressure nor the deoxygenation response in full arterial occlusion, which could have provided a better understanding of the similarities between 60 and $80 \%$ AOP.

\section{CONCLUSION}

Our findings indicate that a relative pressure above 40\% of the AOP at rest seems to be required to potentiate the metabolic stress of low-intensity knee extension exercise. Second, they also provide evidence that with the cuff inflated between sets, there is a hampered recovery of tissue oxygenation and deoxygenation levels between higher and lower BFR pressures. Third, they also demonstrate that $\mathrm{BFR} \geq 60 \%$ of AOP does not enhance oxygen extraction during low-intensity exercise. Fourth, we found an interaction between the magnitude of BFR (from 60 to $80 \%$ ) and the recovery of muscle deoxygenation during the inter-set rest intervals. Thus, while deoxygenated $[\mathrm{HHb}+\mathrm{Mb}]$ remains virtually unchanged during recovery from each set of exercise performed at $60 \%$ AOP, its values actually increase when using $80 \%$ AOP.

\section{ETHICS STATEMENT}

This study was approved by the Faculty's Ethics Committee (CEFMH 17/2014) and in accordance with the Declaration of Helsinki.

\section{AUTHOR CONTRIBUTIONS}

JR, PF, GM, MV, PM-H, and FA conceived and designed the experiments. JR, PF, MV, and JI performed the experiments. JR, PF, JV, JI, and FA analyzed the data. JR, PF, JV, PM-H, and FA interpreted results of research. JR, JV, MV, and PF drafted the manuscript and prepared the tables and figures. JR, PF, GM, JV, MV, JI, PM-H, and FA edited, critically revised the manuscript and approved the final version of manuscript.

\section{FUNDING}

This work was supported by CIPER-Foundation for Science and Technology (FCT), Portugal (UID/DTP/00447/2019) and PTDC/DTP-DES/5714-2014). JR gratefully acknowledges the "Fundação para a Ciência e Tecnologia, Portugal" ("The Foundation for Science and Technology, Portugal") 
for their post-doctoral fellowship award (reference number SFRH/BPD/84315/2012). The results of the present study do not constitute endorsement of the mentioned instruments by the authors or the journal.

\section{REFERENCES}

Brocherie, F., Millet, G. P., and Girard, O. (2015). Neuro-mechanical and metabolic adjustments to the repeated anaerobic sprint test in professional football players. Eur. J. Appl. Physiol. 115, 891-903. doi: 10.1007/s00421-014-3070-z

Cayot, T. E., Lauver, J. D., Silette, C. R., and Scheuermann, B. W. (2014). Effects of blood flow restriction duration on muscle activation and microvascular oxygenation during low-volume isometric exercise. Clin. Physiol. Funct. Imaging 36, 298-305. doi: 10.1111/cpf.12228

Conceição, M. S., Gáspari, A. F., Ramkrapes, A. P. B., Junior, E. M. M., Bertuzzi, R., Cavaglieri, C. R., et al. (2018). Anaerobic metabolism induces greater total energy expenditure during exercise with blood flow restriction. PLoS One 13:e0194776. doi: 10.1371/journal.pone.0194776

Cook, S. B., Clark, B. C., and Ploutz-Snyder, L. L. (2007). ). Effects of exercise load and blood-flow restriction on skeletal muscle function. Med. Sci. Sports Exerc. 39, 1708-1713. doi: 10.1249/mss.0b013e31812383d6

Corrigan, J. H., Burns, J., Huggett, R. J., Mackintosh, A. F., and Mary, D. A. S. G. (2008). The direct effect of leg position on calf blood flow measured by venous occlusion plethysmography. Artery Res. 2, 44-48. doi: 10.1016/j.artres.2007.11.001

Fatela, P., Reis, J. F., Mendonca, G. V., Avela, J., and Mil-Homens, P. (2016). Acute effects of exercise under different levels of blood-flow restriction on muscle activation and fatigue. Eur. J. Appl. Physiol. 116, 985-995. doi: 10.1007/s00421016-3359-1

Fatela, P., Reis, J. F., Mendonca, G. V., Freitas, T. S., Valamatos, M. J., Avela, J., et al. (2018). Acute neuromuscular adaptations in response to low-intensity blood-flow restricted exercise and high-intensity resistance exercise: Are there any differences? J. Strength Cond. Res. 32, 902-910. doi: 10.1519/JSC. 0000000000002022

Ferrari, M., Binzoni, T., and Quaresima, V. (1997). Oxidative metabolism in muscle. Philos. Trans. R. Soc. Lond. B Biol. Sci. 352, 677-683. doi: 10.1098/rstb. 1997.0049

Ferrari, M., Mottola, L., and Quaresima, V. (2004). Principles, techniques, and limitations of near infrared spectroscopy. Can. J. Appl. Physiol. 29, 463-487. doi: 10.1139/h04-031

Ferrari, M., Muthalib, M., and Quaresima, V. (2011). The use of nearinfrared spectroscopy in understanding skeletal muscle physiology: recent developments. Philos. Trans. A Math. Phys. Eng. Sci. 369, 4577-4590. doi: 10.1098/rsta.2011.0230

Ganesan, G., Cotter, J. A., Reuland, W., Cerussi, A. E., Tromberg, B. J., and Galassetti, P. (2015). Effect of blood flow restriction on tissue oxygenation during knee extension. Med. Sci. Sports Exerc. 47, 185-193. doi: 10.1249/MSS. 0000000000000393

Grassi, B., Quaresima, V., Marconi, C., Ferrari, M., and Cerretelli, P. (1999). Blood lactate accumulation and muscle deoxygenation during incremental exercise. J. Appl. Physiol. 87, 348-355. doi: 10.1152/jappl.1999.87.1.348

Iida, H., Takano, H., Meguro, K., Asada, K., Oonuma, H., Morita, T., et al. (2005). Hemodynamic and autonomic nervous responses to the restriction of femoral blood flow by KAATSU. Int. J. KAATSU Train. Res. 1, 57-64. doi: 10.3806/ijktr.1.57

Karabulut, M., Leal, J. A., Garcia, S. D., Cavazos, C., and Bemben, M. (2014). Tissue oxygenation, strength and lactate response to different blood flow restrictive pressures. Clin. Physiol. Funct. Imaging 34, 263-269. doi: 10.1111/cpf.12090

Kilgas, M. A., McDaniel, J., Stavres, J., Pollock, B. S., Singer, T. J., and Elmer, S. J. (2018). Limb blood flow and tissue perfusion during exercise with blood flow restriction. Eur. J. Appl. Physiol. 119, 377-387. doi: 10.1007/s00421-018-4029-2

Lauver, J. D., Cayot, T. E., Rotarius, T., and Scheuermann, B. W. (2017). The effect of eccentric exercise with blood flow restriction on neuromuscular activation, microvascular oxygenation, and the repeated bout effect. Eur. J. Appl. Physiol. 117, 1005-1015. doi: 10.1007/s00421-017-3589-x

\section{ACKNOWLEDGMENTS}

The authors thank the participants for their dedication, commitment, and cooperation with this study.

Lixandrão, M. E., Ugrinowitsch, C., Laurentino, G., Libardi, C. A., Aihara, A. Y., Cardoso, F. N., et al. (2015). Effects of exercise intensity and occlusion pressure after 12 weeks of resistance training with blood-flow restriction. Eur. J. Appl. Physiol. 115, 2471-2480. doi: 10.1007/s00421-015-3253-2

Loenneke, J. P., Fahs, C. A., Wilson, J. M., and Bemben, M. G. (2011). Blood flow restriction: the metabolite/volume threshold theory. Med. Hypotheses 77, 748-752. doi: 10.1016/j.mehy.2011.07.029

Loenneke, J. P., Kim, D., Fahs, C. A., Thiebaud, R. S., Abe, T., Larson, R. D., et al. (2015). The effects of resistance exercise with and without different degrees of blood-flow restriction on perceptual responses. J. Sports Sci. 33, 1472-1479. doi: 10.1080/02640414.2014.992036

Loenneke, J. P., Thiebaud, R. S., and Abe, T. (2014a). Does blood flow restriction result in skeletal muscle damage? A critical review of available evidence. Scand. J. Med. Sci. Sports 24, e415-e422. doi: 10.1111/sms.12210

Loenneke, J. P., Thiebaud, R. S., Abe, T., and Bemben, M. G. (2014b). Blood flow restriction pressure recommendations: the hormesis hypothesis. Med. Hypotheses 82, 623-626. doi: 10.1016/j.mehy.2014.02.023

Loenneke, J. P., Thiebaud, R. S., Fahs, C. A., Rossow, L. M., Abe, T., and Bemben, M. G. (2013). Blood flow restriction does not result in prolonged decrements in torque. Eur. J. Appl. Physiol. 113, 923-931. doi: 10.1007/s00421-012-2502-x

Loenneke, J. P., Wilson, J. M., Marín, P. J., Zourdos, M. C., and Bemben, M. G. (2012). Low intensity blood flow restriction training: a meta-analysis. Eur. J. Appl. Physiol. 112, 1849-1859. doi: 10.1007/s00421-011-2167-x

Manini, T. M., and Clark, B. C. (2009). Blood flow restricted exercise and skeletal muscle health. Exerc. Sport Sci. Rev. 37, 78-85. doi: 10.1097/JES. 0b013e31819c2e5c

Mendonca, G. V., Vaz, J. R., Pezarat-Correia, P., and Fernhall, B. (2015). Effects of walking with blood flow restriction on excess post-exercise oxygen consumption. Int. J. Sports Med. 36, e11-e18. doi: 10.1055/s-0034-1395508

Mouser, J. G., Laurentino, G. C., Dankel, S. J., Buckner, S. L., Jessee, M. B., Counts, B. R., et al. (2017). Blood flow in humans following low-load exercise with and without blood flow restriction. Appl. Physiol. Nutr. Metab. 42, 1165-1171. doi: 10.1139/apnm-2017-0102

Neto, G. R., Santos, H. H., Sousa, J. B. C., Júnior, A. T. A., Araújo, J. P., Aniceto, R. R., et al. (2014). Effects of high-intensity blood flow restriction exercise on muscle fatigue. J. Hum. Kinet. 41, 163-172. doi: 10.2478/hukin-2014-0044

Patterson, S. D., and Ferguson, R. A. (2010). Increase in calf post-occlusive blood flow and strength following short-term resistance exercise training with blood flow restriction in young women. Eur. J. Appl. Physiol. 108, 1025-1033. doi: 10.1007/s00421-009-1309-x

Pereira, M. I. R., Gomes, P. S. C., and Bhambhani, Y. N. (2007). A brief review of the use of near infrared spectroscopy with particular interest in resistance exercise. Sports Med. 37, 615-624. doi: 10.2165/00007256-20073707000005

Perrey, S., and Ferrari, M. (2018). Muscle oximetry in sports science: a systematic review. Sports Med. 48, 597-616. doi: 10.1007/s40279-017-0820-1

Pope, Z. K., Willardson, J. M., and Schoenfeld, B. J. (2013). Exercise and blood flow restriction. J Strength Cond. Res. 27, 2914-2926. doi: 10.1519/JSC. 0b013e3182874721

Rovati, L., Bandera, A., Donini, M., Salvatori, G., and Pollonini, L. (2004). Design and performance of a wide-bandwidth and sensitive instrument for nearinfrared spectroscopic measurements on human tissue. Rev. Sci. Instrum. 75, 5315-5325. doi: 10.1063/1.1818588

Schoenfeld, B. J. (2010). The mechanisms of muscle hypertrophy and their application to resistance training. J. Strength Cond. Res. 24, 2857-2872. doi: 10.1519/JSC.0b013e3181e840f3

Scott, B. R., Loenneke, J. P., Slattery, K. M., and Dascombe, B. J. (2015). Exercise with blood flow restriction: an updated evidence-based approach for enhanced muscular development. Sports Med. 45, 313-325. doi: 10.1007/s40279-0140288-1 
Slysz, J., Stultz, J., and Burr, J. F. (2016). The efficacy of blood flow restricted exercise: a systematic review \& meta-analysis. J. Sci. Med. Sport 19, 669-675. doi: 10.1016/j.jsams.2015.09.005

Soares, R. N., McLay, K. M., George, M. A., and Murias, J. M. (2017). Differences in oxidative metabolism modulation induced by ischemia/reperfusion between trained and untrained individuals assessed by NIRS. Physiol. Rep. 5:e13384. doi: $10.14814 /$ phy2.13384

Suga, T., Okita, K., Takada, S., Omokawa, M., Kadoguchi, T., Yokota, T., et al. (2012). Effect of multiple set on intramuscular metabolic stress during lowintensity resistance exercise with blood flow restriction. Eur. J. Appl. Physiol. 112, 3915-3920. doi: 10.1007/s00421-012-2377-x

Takarada, Y., Nakamura, Y., Aruga, S., Onda, T., Miyazaki, S., and Ishii, N. (2000). Rapid increase in plasma growth hormone after low-intensity resistance exercise with vascular occlusion. J. Appl. Physiol. 88, 61-65. doi: 10.1152/jappl. 2000.88.1.61

Takarada, Y., Sato, Y., and Ishii, N. (2002). Effects of resistance exercise combined with vascular occlusion on muscle function in athletes. Eur. J. Appl. Physiol. 86, 308-314. doi: 10.1007/s00421-001-0561-5

Van Beekvelt, M. C. P., Colier, W. N. J. M., Wevers, R. A., and Van Engelen, B. G. M. (2001). Performance of near-infrared spectroscopy in measuring local O 2 consumption and blood flow in skeletal muscle. J. Appl. Physiol. 90, 511-519. doi: 10.1152/jappl.2001.90.2.511
Willis, S. J., Alvarez, L., Millet, G. P., and Borrani, F. (2017). Changes in muscle and cerebral deoxygenation and perfusion during repeated sprints in hypoxia to exhaustion. Front. Physiol. 8:846. doi: 10.3389/fphys.2017.00846

Yanagisawa, O., and Sanomura, M. (2017). Effects of low-load resistance exercise with blood flow restriction on high-energy phosphate metabolism and oxygenation level in skeletal muscle. Interv. Med. Appl. Sci. 9, 67-75. doi: 10.1556/1646.9.2017.16

Yasuda, T., Brechue, W. F., Fujita, T., Sato, Y., and Abe, T. (2008). Muscle activation during low-intensity muscle contractions with varying levels of external limb compression. J. Sports Sci. Med. 7, 467-474.

Conflict of Interest Statement: The authors declare that the research was conducted in the absence of any commercial or financial relationships that could be construed as a potential conflict of interest.

Copyright $\odot 2019$ Reis, Fatela, Mendonca, Vaz, Valamatos, Infante, Mil-Homens and Alves. This is an open-access article distributed under the terms of the Creative Commons Attribution License (CC BY). The use, distribution or reproduction in other forums is permitted, provided the original author(s) and the copyright owner(s) are credited and that the original publication in this journal is cited, in accordance with accepted academic practice. No use, distribution or reproduction is permitted which does not comply with these terms. 